\title{
CLINICAL PROFILE AND SHORT TERM OUTCOME OF HYPOXIC ISCHEMIC ENCEPHALOPATHY AMONG BIRTH ASPHXIATED BABIES IN A TERTIARY CARE HOSPITAL
}

\author{
Hafsa Niaz, Jawad Jalil*, Qamar Uz Zaman, Khan, Faisal Basheer*, Shahzad Akhtar*, Naila Hamid \\ Pak Emirates Military Hospital/National University of Medical Sciences (NUMS) Rawalpindi Pakistan, *Combined Military \\ Hospital/National University of Medical Sciences (NUMS) Rawalpindi Pakistan
}

\begin{abstract}
Objective: To determine the clinical profile, selected antepartum and intrapartum risk factor for adverse short term outcomes of hypoxic ischemic encephalopathy in babies with birth asphyxia.

Study Design: Cross sectional study.

Place and Duration of Study: Neonatal Intensive Care Unit of Pak Emirates Military Hospital, Rawalpindi, from Jan to Dec 2018.

Methodology: This study including all birth asphyxiated babies born who fulfilled the inclusion criteria. Following data was collected prospectively regarding gender, gestational age, birth weight and mode of delivery, maternal age, antenatal follow up, history of premature rupture of membranes and meconium stained liquor. Babies were categorized into different stages of hypoxic ischemic encephalopathy according to Sarnat and Sarnat staging. Selected antepartum and intrapartum risk factors leading to hypoxic insult at birth were studied and short-term outcome was recorded in the form of need of mechanical ventilation, mortality and discharge from the hospital.

Results: The frequency of birth asphyxia turned out to be $122 / 5986(2.03 \%)$ at our center. Thirty four $(27.87 \%)$ required mechanical ventilation, mortality was recorded at 20/122 (16.39\%). While $61(50 \%)$ babies suffered from stage I hypoxic ischemic encephalopathy, 13/20 (65\%) of newborn who expired were suffering from grade III hypoxic ischemic encephalopathy.

Conclusion: The severity of hypoxic ischemic encephalopathy affects the outcome of newborns having birth asphyxia with hypoxic ischemic encephalopathy grade III associated with maximum mortality. Early identifycation of pregnancies at risk for asphyxia, with appropriate intervention in selected cases is the key to prevent birth asphyxia and its ensuing neonatal complications.
\end{abstract}

Keywords: Birth asphyxia, Hypoxic ischemic encephalopathy, Mechanical ventilation.

\footnotetext{
This is an Open Access article distributed under the terms of the Creative Commons Attribution License (http://creativecommons.org/licenses/by/4.0), which permits unrestricted use, distribution, and reproduction in any medium, provided the original work is properly cited.
}

\section{INTRODUCTION}

Birth asphyxia is defined by the World Health Organization as "the failure to initiate and sustain breathing at birth"1. According to WHO, 4-9 million newborns develop birth asphyxia each year and at least the same number develop severe consequences such as epilepsy, cerebral palsy and developmental delay $^{2}$. While $3 \%$ of all infants in developing countries suffer from moderate to severe birth asphyxia, the mortality rate in developed world is only $<0.1 \%^{3}$. This low mortality in developed world is due to improved primary obstetric care. Birth asphyxia is a global issue especially in developing countries like Pakistan. Inci-

Correspondence: Dr Hafsa Niaz, Department of Paediatrics, Pak Emirates Military Hospital, Rawalpindi Pakistan

Received: 05 Feb 2020; revised received: 06 May 2020; accepted: 08 May 2020 dence of perinatal asphyxia in Pakistan is about $3.3 \%^{4}$.

Birth asphyxia can be caused by events that have their roots in either the antepartum, the intra partum or the postpartum periods or combination thereof ${ }^{2}$. Common risk factors include increasing or decreasing maternal age, non-attendance for antenatal care, low birth weight infants, malpresentation, prolonged rupture of membranes, meconium staining, maternal anemia, vaginal bleeding, maternal fever at the time of delivery, mode of delivery after prolonged second stage of labor like normal vertex delivery, caesarian section and multiple births ${ }^{5,6}$.

Sarnat and Sarnat proposed a staging system useful in classifying hypoxic ischemic encephalopathy, according to which stages 1, 2 and 3 correlates with the description of mild, moderate and severe encephalopathy ${ }^{7}$. Though the topic has been extensively stu- 
died and reviewed worldwide, limited local data is available and there is little change in the outcome of perinatal asphyxia. One reason is that we do not know specifically the crucial risk factors that would assist to intervene both prenatally and postnatally to prevent HIE, which may follow intrapartum asphyxia. Nor do we know how to identify the neonates with asphyxia who are the greatest risk of developing encephalopathy ${ }^{8}$. This study was conducted to determine the clinical profile, selected antepartum and intrapartum risk factors leading to birth asphyxia and record their short-term outcome in the form of grades of hypoxic ischemic encephalopathy, mechanical ventilation, mortality and discharge from the hospital.

\section{METHODOLOGY}

This was a prospective cross sectional study which was conducted from January 2018 to December 2018 at the Neonatal Intensive Care Unit of PEMH Rawalpindi. Ethical approval was sought from the ethical committee PEMH Rawalpindi letter number A/28/10 dated 8 Aug 2019. A total of 122 cases of birth asphyxia that fulfilled the inclusion and exclusion criteria were included in the study through random sampling technique. All newborn born in hospital regardless of mode of delivery with gestational age between 34 weeks to 40 weeks and evidence of birth asphyxia were included in the study. Birth asphyxia was defined as delayed first cry for 5 minutes or APGAR score of 5 at 5 minutes of age with no improvement to more than $7 / 10$ at 20 minutes of age. Only APGAR score was used to define asphyxia.

Newborn with gross dysmorphism, congenital heart defects, chromosomal abnormalities, congenital infections, infectious and metabolic causes of central nervous system encephalopathy, outdoor deliveries and extremely low birth weight (birth weight less than $1000 \mathrm{~g}$ ) were excluded from the study. All the indoor deliveries were attended by pediatricians. Neonatal resuscitation if needed was performed as per the neonatal resuscitation protocol (NRP).

After obtaining the informed consent newborns were registered in the study. The newborns were followed during their stay in NICU, parameters recorded were; gender, gestational age, weight and mode of delivery. Babies were examined in detail and the severity of hypoxic ischemic encephalopathy was graded according to Sarnat and Sarnat staging7. Depending on the neurobehavioral signs neonates were divided into three stages1, 2 and 3. Only clinical para- meters of Sarnat and Sarnat staging were used as there was no facility to perform EEG of neonates in NICU.

Risk factors occurring before childbirth, with reference to the mother were labelled antepartum risk factors they included maternal age (18-27 and28-37 years) and compliance to antenatal follow up. Risk factors which occurred during labor and delivery were called intrapartum risk factorswhich were premature rupture of membranes and meconium stained liquor. Maternal data for only selected risk factors was obtained.

Short-term outcome was recorded in the form of need of mechanical ventilation, mortality and discharge from the hospital. Descriptive statistics were calculated for variables. All the data was analyzed on SPSS 24 for windows.

\section{RESULTS}

A total of 5986 patients were delivered, out of which the frequency of birth asphyxia turned out to be $122 / 5986(2.03 \%)$. Out of 122 patients who suffered from birth asphyxia the mortality was recorded at 20 (16.39\%). Eighty $(65.57 \%)$ were male while 42 (34.43\%) were females. Mortality was found to be higher in male patients i.e. $13(65 \%)$ as compared to female patients i.e. 7 (35\%). Seventy Nine $(64.75 \%)$ had normal birth weight, 36 (29.51\%) were low birth weight (LBW), 3 (4.92\%) had very low birth weight (VLBW), $17(85 \%)$ patients who died had normal birth weight.

Table-I: Characteristics of newborn.

\begin{tabular}{|c|c|c|}
\hline Gender & n (\%) & $\begin{array}{c}\text { Mortality } \\
(n=20)\end{array}$ \\
\hline Male & $80(65.57 \%)$ & $13(65 \%)$ \\
\hline Female & $42(34.43 \%)$ & $7(35 \%)$ \\
\hline \multicolumn{3}{|l|}{ Birth Weight } \\
\hline Normal & $79(64.75 \%)$ & $17(85 \%)$ \\
\hline LBW & $36(29.51 \%)$ & $2(10 \%)$ \\
\hline VLBW & $3(4.92 \%)$ & $1(5 \%)$ \\
\hline \multicolumn{3}{|l|}{ Gestational Age } \\
\hline$>37$ weeks & $84(68.85 \%)$ & $13(65 \%)$ \\
\hline$<36+6$ weeks & $38(31.15 \%)$ & $7(35 \%)$ \\
\hline \multicolumn{3}{|l|}{ Mode of Delivery } \\
\hline $\begin{array}{l}\text { Spontaneous } \\
\text { Vertex Delivery }\end{array}$ & $61(50 \%)$ & $10(50 \%)$ \\
\hline $\begin{array}{l}\text { Lower Segment } \\
\text { Caesarian Section }\end{array}$ & $61(50 \%)$ & $10(50 \%)$ \\
\hline
\end{tabular}

Eighty four $(68.85 \%)$ were term babies while 38 $(31.15 \%)$ were preterm. Fifty percent were delivered via SVD while an equal number were born by LSCS. 
The characteristics of newborns with birth asphyxia with frequency were shown in table-I. Sixty three $(51.63 \%)$ lied in age group 18-27 years, 59 (48.36\%) had ages between 28-37 years. Mortality was found to be higher in 28-37 year old female. Eighty six (70.49\%) had regular antenatal visits while 36 (29.51\%) had no antenatal visits. Nine (45\%) babies expired whose mothers had no antenatal visits. Antepartum risk factors were shown in table-II.

Table-II: Ante partum risk factors.

\begin{tabular}{|c|c|c|c|c|}
\hline \multicolumn{2}{|c|}{ Maternal Age } & \multicolumn{2}{|c|}{ n (\%) } & Mortality \\
\hline \multicolumn{2}{|c|}{$18-27$ years } & \multicolumn{2}{|c|}{$63(51.63 \%)$} & $5(25 \%)$ \\
\hline \multicolumn{2}{|c|}{$28-37$ years } & \multicolumn{2}{|c|}{$59(48.36 \%)$} & $15(75 \%)$ \\
\hline \multicolumn{5}{|c|}{ Antenatal Follow-Up } \\
\hline \multicolumn{2}{|c|}{ Yes } & \multicolumn{2}{|c|}{$86(70.49 \%)$} & $11(55 \%)$ \\
\hline \multicolumn{2}{|l|}{ No } & \multicolumn{2}{|c|}{$36(29.51 \%)$} & $9(45 \%)$ \\
\hline \multicolumn{5}{|c|}{ Table-III: Intrapartum risk factors. } \\
\hline \multicolumn{2}{|c|}{$\begin{array}{l}\text { Premature Rupture } \\
\text { of Membranes }\end{array}$} & \multicolumn{2}{|r|}{ n (\%) } & Mortality \\
\hline \multicolumn{2}{|c|}{ Yes } & \multicolumn{2}{|c|}{$30(24.59 \%)$} & $6(30 \%)$ \\
\hline \multicolumn{2}{|l|}{ No } & \multicolumn{2}{|c|}{$92(75.41 \%)$} & $14(70 \%)$ \\
\hline \multicolumn{5}{|c|}{ Meconium Stained Liquor } \\
\hline \multicolumn{2}{|c|}{ Yes } & \multicolumn{2}{|c|}{$25(20.49 \%)$} & $4(20 \%)$ \\
\hline \multicolumn{2}{|l|}{ No } & \multicolumn{2}{|c|}{$97(79.51 \%)$} & $16(80 \%)$ \\
\hline \multicolumn{5}{|c|}{ Table-IV: Outcome of newborns. } \\
\hline \multicolumn{4}{|c|}{ Mechanical Ventilation } & n (\%) \\
\hline \multicolumn{2}{|c|}{ Yes } & \multicolumn{3}{|c|}{$34(27.87 \%)$} \\
\hline \multicolumn{2}{|c|}{ No } & \multicolumn{3}{|c|}{$88(72.13 \%)$} \\
\hline \multicolumn{5}{|c|}{ HIE Grades } \\
\hline \multicolumn{2}{|c|}{ Grade I } & & & $(50 \%)$ \\
\hline Grade & & & 42 & $(34.43 \%)$ \\
\hline Grade & & & & $(15.57 \%)$ \\
\hline Outcom & & & & \\
\hline Dischar & & & 102 & $(83.61 \%)$ \\
\hline Expirec & & & \begin{tabular}{l|l|} 
& 20 \\
\end{tabular} & $(16.39 \%)$ \\
\hline Table-V & Outcome ac & ord & ing to HIE S & tages. \\
\hline $\begin{array}{l}\text { HIE } \\
\text { Grade }\end{array}$ & $\begin{array}{c}\text { Ventilated } \\
(\%)\end{array}$ & & $\begin{array}{c}\text { ischarged } \\
(\%)\end{array}$ & Expired $(\%)$ \\
\hline I & $7(20 \%)$ & & $9(57.84 \%)$ & $2(10 \%)$ \\
\hline II & $11(32 \%)$ & & $7(36.27 \%)$ & $5(25 \%)$ \\
\hline III & $16(47 \%)$ & & $6(5.88 \%)$ & $13(65 \%)$ \\
\hline $\begin{array}{l}\text { Total } \\
122\end{array}$ & $34(27.87 \%)$ & & $2(83.61 \%)$ & $20(16.39 \%)$ \\
\hline
\end{tabular}

Thirty $(24.59 \%)$ babies with birth asphyxia were born to mothers with history of premature rupture of membranes and $25(20.49 \%)$ had history of meconium stained liquor. Intrapartum risk factors recorded were shown in table $3.34(27.87 \%)$ babies with birth asphyxia required mechanical ventilation. Sixty one $(50 \%)$ had HIE grade I, $42(34.43 \%)$ had HIE grade II while $19(15.57 \%)$ had HIE grade III. Out of 122 patients with birth asphyxia $102(83.61 \%)$ were discharged with stable signs and $20(16.39 \%)$ expired. Outcomes of newborn were shown in table-IV.

In 13/20 (65\%) newborn who expired were suffering from hypoxic ischemic encephalopathy (HIE) grade III. Sixteen (47\%) patients with HIE III required mechanical ventilation. Outcomes in hypoxic ischemic encephalopathy according to stages were mentioned in table-V.

\section{DISCUSSION}

This was a single center study conducted at the Neonatal Intensive Care Unit of PEMH Rawalpindi. It was aimed at finding out clinical characteristics of babies born with hypoxic insult at birth due to various antepartum and intrapartum causes, classify them according to Sarnat and Sarnat staging and study their short-term outcomes in terms of need of mechanical ventilation, mortality and discharge. The frequency of birth asphyxia turned out to be 122/5986 (2\%).

Our study documents that $80(65.57 \%)$ patients who suffered from birth asphyxia were males. Although other studies also show male predominance but the number of males in our study were a lot more as compared to other studies ${ }^{3,9}$ with male to female ratio of 2:1. Mortality was also found to be higher in male babies. Thirty-one percent of the neonates with birth asphyxia were preterm deliveries. Mortality was found to be higher in term babies as compared to preterm term babies ( $65 \%$ vs. $35 \%)$ in our study whereas according to a recent study of asphyxiated babies in Nepal, prematurity is one of the major risk factor adversely affecting the outcomes in birth asphyxia babies ${ }^{10}$. There was no significant relationship between birth weight and asphyxia, 79 (64\%) babies had normal birth weight while 36 (29\%) were low birth weight $(>2.5 \mathrm{~kg})$ and $3(4.9 \%)$ had very low birth weight $(>1.5 \mathrm{~kg})$. Low birth weight is identified as one of the major culprits for causing birth asphyxia in international studies ${ }^{11}$.

The age of mother at delivery was found to have significant effect on the outcome of babies in terms of mortality, in our study. Fifty one percent of mothers were between 18 to 27 years of age and $48 \%$ lied in 28 to 37 years age group. Fifteen (75\%) babies who expired belonged to mothers with 28 to 37 years age group. This was similar to the results reported from another study from Sweden ${ }^{12}$. As reported in previous 
studies maternal age more than 35 is a risk factor associated with birth asphyxia. Eighty six $(70 \%)$ patients had regular antenatal follow up while $36(29 \%)$ had no or poor antenatal follow up. Nine $(45 \%)$ out of 20 patients who expired were born to mothers who had no antenatal checkup during their pregnancy.

In our study the number of birth asphyxia babies delivered via spontaneous vertex delivery was equal to lower segment caesarian section. No effect on outcome of babies was seen with regards to mode of delivery. Increased rate of caesarian section shows skill of detecting perinatal asphyxia in a tertiary care hospital and going for early intervention. In other studies like Chishty ${ }^{13}$ the proportion of birth asphyxia was predominantly greater in spontaneous vertex delivery (SVD) in indoor cases, but Zulfiqar ${ }^{14}$ in his study described $66 \%$ of babies delivered by caesarian section had birth asphyxia as compared to $34 \%$ cases born by SVD.

The frequency of birth asphyxia in patients suffering from premature rupture of membranes (PROM) turned out to be $24.9 \%$. This was found to be same as seen by another national study by Majeed et al ${ }^{6}$ where he showed that $24 \%$ babies whose mother had PROM, developed birth asphyxia. PROM is not only associated with increased incidence of emergency caesarian section but it is also associated with birth asphyxia but the frequency of birth asphyxia in PROM was found to be higher as compared to international studies $(24.9 \%$ vs. $10 \%)^{15}$.

Meconium stained liquor has been described as one of the most frequent risk factor associated with birth asphyxia ${ }^{16}$. Although the frequency of meconium stained liquor in birth asphyxia patients is reported higher in the international studies, it was found in 25\% of our patients. Out of the 20 babies who expired 15 $(75 \%)$ babies had a history of meconium stained liquor. Meconium stained liquor has strongly been associated with fetal distress in various studies ${ }^{17}$. Every seventh pregnancy suffers from meconium stained liquor and the frequency is increased in term and post term deliveries, $10-20 \%$ and $30-40 \%$ respectively. It is documented in $4 \%$ of deliveries less than 37 weeks of gestation $^{17}$.

The mechanical ventilation rates in birth asphyxia patients was found to be $27.87 \%$. Seven $(20 \%)$ patients with HIE grade I were ventilated, $11(32 \%)$ with HIE grade II while all the neonates with grade III hypoxic ischemic insult required mechanical ventilation. All the 20 babies who expired required mechanical ventilation. Birth asphyxia is one of the most common indication of mechanical ventilation in neonatal age group $(18 \%)^{18}$. In our study $34(27.87 \%)$ patients with birth asphyxia required mechanical ventilation which is higher as compared to international studies. Mechanical ventilation adds further risk to the mortality of newborns with 33\% mortality in birth asphyxia patients $^{18}$. Fifty percent of the babies suffered from hypoxic ischemic encephalopathy grade I while the frequency of grade II and grade III was found to be $34.5 \%$ and $15.5 \%$ respectively. In our study majority of babies suffered from grade I HIE whereas other studies from Pakistan document the predominance of HIE $\mathrm{II}^{19}$. Regional studies also document the predominance of HIE I ${ }^{19}$. The severity of HIE affects the outcome. HIE grade III is associated with $100 \%$ death or disability and the survivors usually die by 5 years of age due to various causes. In our study the $16.3 \%$ of the babies died due to birth asphyxia. Maximum mortality was documented in babies who suffered from HIE grade III as compared to HIE grade II and grade I (65\% vs. $25 \%$ and $10 \%)$.

The study was conducted at Neonatal Intensive Care Unit where all the patients were delivered under the care of classified obstetricians and attended by qualified pediatricians. Cases of birth asphyxia were identified right after birth and staged accordingly. They were managed and followed up till discharge or mortality. However our study underestimates the actual mortality and morbidity of birth asphyxia in Pakistan. Maternal comorbidities like anemia, hypertension and diabetes were not studied which are predisposing risk factors for birth asphyxia ${ }^{20,21}$.

Our local data is very limited as regards to the risk factors of birth asphyxia, hence we need to conduct various multicenter studies in different areas of Pakistan to evaluate the risk factors of birth asphyxia so that interventions can be done to educate and guide the masses regarding this very important issue and implement appropriate management strategies ${ }^{22}$. Our study opens the door of large proportion of research which needs on this topic to define more accurately the true burden of birth asphyxia in developing countries and to discuss the contribution of common risk factors like maternal malnutrition and infections on the outcome of asphyxia ${ }^{22}$.

\section{CONCLUSION}

The severity of HIE affects the outcome of newborns having birth asphyxia, with HIE grade III associated with maximum mortality. Early identification 
of pregnancies at risk for asphyxia, with appropriate intervention in selected cases is the key to prevent birth asphyxia and its ensuing neonatal complications.

\section{CONFLICT OF INTEREST}

This study has no conflict of interest to be declared by any author.

\section{REFERENCES}

1. World Health Organization (WHO). Basic Newborn Resuscitation: A Practical Guide. Geneva, Switzerland: World Health Organization; 1997.

2. Haider BA, Bhutta ZA. Birth asphyxia in developing countries: current status and public health implications. Curr Probl Pediatr Adolesc Health Care 2006; 5(36): 178-88.

3. Amritanshu K, Smriti S, Kumar V, Pathak A, Banerjee DP. Clinical profile and short-term outcome of hypoxic ischemic encephalopathy among birth asphyxiated babies in Katihar medical college hospital. J Clin Neonatol 2014; 3(4): 195-99.

4. Maqbool S, Hodge W. Birth asphyxia. in: handbook of neonatal care. Lahore: Nabiza publishers 1996; 80-88.

5. Butt TK, Farooqui R, Khan MA. Risk factors for hypoxic encephlopathy in children. J Coll Physicians Surg Pak 2008; 18(7): 428-32.

6. Majeed R, Memon Y, Majeed F, Shaikh NP, Rajar UD. Risk factors of birth asphyxia. J Ayub Med Coll Abbottabad 2007; 19(3): 67-71.

7. Douglas-Escobar M, Weiss MD. Hypoxic-ischemic encephalopathy: a review for the clinician. J Am Med Assoc Pediatr 2015; 169(4): 397-403.

8. Perlman JM. Markers of asphyxia and neonatal brain injury. $\mathrm{N}$ Engl J Med 1999; 341(5): 364-65.

9. Ibrahim NA, Muhye A, Abdulie S. Prevalence of birth asphyxia and associated factors among neonates delivered in Dilchora Referral Hospital. Dire Dawa, East Ethiop Clin Mother Child Heal 2017; 14(279): 2-9.

10. Lundgren C, Brudin L, Wanby AS, Blomberg M. Ante-and intra- partum risk factors for neonatal hypoxic ischemic encephalopathy. J Matern Neonatal Med 2018; 31(12): 1595-601.

11. Pitsawong $C$, Panichkul P. Risk factors associated with birth asphyxia in Phramongkutklao Hospital. Thai J Obstet Gynaecol 2012; 19(4): 165-71.

12. Milsom I, Ladfars L, Thiringer K. Influence of maternal, obstetric and fetal risk factors on the prevalence of birth asphyxia at termin, a Swedish urban population. Acta Obstet Gynecol Scand 2002; 81(10): 909-13.

13. Chishty AL, Iqbal MA, Anjum A, Maqbool S. Risk factors analysis of birth asphyxia at the children's hospital Lahore. Pak Paed J 2002; 26(2): 47-53.

14. Zulfiqar R, Naeemullah S. Severity of hypoxic ischaemic encephalopathy in neonates with birth asphyxia. J Rawal Med Coll 2007; 11(1): 18-22.

15. Rajan R, Menon V. Preterm premature rupture of membranes: correlates and pregnancy outcome in a tertiary care setting. Int J Res Med Sci 2016; 4(8): 3310-16.

16. Ross MG. Meconium aspiration syndrome more than intrapartum meconium. Mass Medical Soc N Engl J Med 2005; 353: 946-48.

17. Prakash KP, BK SD. Respiratory distress in vigorous babies born through meconium stained amniotic fluid: incidence, onset, risk factors and predictors at birth. Int J Contemp Pediatr 2017; 4(2): 390.

18. Iqbal Q, Younus MM, Ahmed A, Ahmad I, Iqbal J, Charoo BA, et al. Neonatal mechanical ventilation: Indications and outcome. Indian Soc Crit Care Med 2015; 19(9): 523-29.

19. Malik AR, Quddusi AI, Fatima N, Iqbal I, Javeed AM. Full term babies. Prof Med J 2017; 24(06): 828-33.

20. Kaye D. Antenatal and intrapartum risk factors for birth asphyxia among emergency obstetric referrals in Mulago hospital, Kampala, Uganda. East Afr Med J 2003; 80(3): 140-43.

21. Mbweza E. Risk factors for perinatal asphyxia at Queen Elizabeth Central Hospital, Malawi. Clin Excell Nurse Pract 2000; 4(3): 158-62.

22. Aslam HM, Saleem S, Afzal R, Iqbal U, Saleem SM, Abid MW, et al. Risk factors of birth asphyxia. Ital J Pediatr 2014; 40(1): 94. 